\title{
PredictC, liquid biopsy in precision oncology: a technology transfer experience in the Spanish health system.
}

Lorena Alonso-Alconada1, Jorge Barbazan', Sonia Candamio', Josep Lluis Falco², Cristina Anton ${ }^{3}$, Carlos Martin ${ }^{3}$, Gustavo Fuster ${ }^{4}$, Mabel Sampedro5, Carlos Grande $^{6}$, Ruben Lado ${ }^{6}$, Laura Sampietro-Colom7, Eladio $\mathrm{Crego}^{8}$, Sergio Figueiras ${ }^{9}$, Luis Leon-Mateos ${ }^{9}$, Rafael Lopez-Lopez ${ }^{1}$, Miguel Abal ${ }^{1}$

1 Translational Medical Oncology - CIBERONC, Health Research Institute of Santiago (IDIS). University Hospital of Santiago (SERGAS), Santiago de Compostela, Spain.

2 Antares Consulting, Barcelona, Spain.

3 UETeS, Universidad Francisco de Vitoria, Madrid, Spain.

4 Hoffmann Eitle, Madrid, Spain.

${ }^{5}$ Department of Innovation and Transfer, Ramon Dominguez Foundation, Santiago de Compostela, Spain.

${ }^{6}$ Medical and Health Technology Innovation Platform (ITEMAS) - Galician Network, Santiago de Compostela, Spain.

7 Health Technology Assessment Unit, Clinic Hospital, Barcelona, Spain.

${ }^{8}$ EFT Consulting, Spain

${ }^{9}$ Health Knowledge Agency (ACIS), Galician Health System (SERGAS), Santiago de Compostela, Spain.

\section{Keywords:}

Liquid biopsy; metastatic colorectal cancer; regulatory roadmap; benchmarking; product development; intellectual property; cost-effectiveness; expert panel.

Corresponding autor: Miguel Abal, Translational Medical Oncology - CIBERONC (IDIS/SERGAS); Trav. Choupana s/n 15706; Santiago de Compostela (Spain); Email: miguel.abal.posada@sergas.es

Financial support: Pre-commercial Development of Research Results Program (PRIS), from the Galician Health System (SERGAS); "la Caixa" Banking Foundation call Caixalmpulse 2015.

\section{Compliance with Ethical Standards:}

The authors declare no conflict of interest. Code of Institutional Ethics Committee: 2014/464; informed consent were signed by all patients. 


\section{Abstract}

Purpose: Management of metastatic disease in oncology includes monitoring of therapy response principally by imaging techniques like CT-Scan. In addition to some limitations, the irruption of liquid biopsy and its application in personalized medicine has encouraged the development of more efficient technologies for prognosis and follow-up of patients in advanced disease.

Methods: PrediCTC constitutes a panel of genes for the assessment of Circulating Tumor Cells (CTC) in metastatic colorectal cancer patients, with demonstrated improved efficiency compared to CT-Scan for the evaluation of early therapy response in a multicenter prospective study. In this work, we designed and developed a technology transfer strategy to define the market opportunity for an eventual implementation of PrediCTC in the clinical practice.

Results: This included the definition of the regulatory framework, the analysis of the regulatory roadmap needed for CE mark, a benchmarking study, the design of a product development strategy, a revision of intellectual property, a cost-effectiveness study and an expert panel consultation.

Conclusion: The definition and analysis of an appropriate technology transfer strategy and the correct balance among regulatory, financial and technical determinants are critical for the transformation of a promising technology into a viable technology, and for the decision of implementing liquid biopsy in the monitoring of therapy response in advanced disease. 


\section{Introduction:}

Metastasis represents the most devastating event in oncology. The presence of locoregional and distant metastasis represents a contraindication to surgery and radiotherapy, is associated with chemotherapy resistance, and is responsible for more than $90 \%$ of cancer related deaths. Colorectal cancer (CRC) is the third most common cancer worldwide being responsible for $8 \%$ of cancer-related deaths in men and $9 \%$ in women [1]. About $80 \%$ of patients with $\mathrm{CRC}$ present with localized or locoregional and resectable disease at diagnosis, with a $90 \%$ five-year relative survival rate in localized disease and $70 \%$ with regional dissemination. Five-year survival rate drops to $13 \%$ for the remaining $20 \%$ patients presenting with distant metastasis at diagnosis [1]. In addition, the risk of relapse also depends on the pathological stage of the primary tumor (30\% for stage II and $50 \%$ for stage III) and is higher within the first two years after surgery. Liver constitutes the main metastatic organ in CRC. Although less frequent, abdominal lymph nodes, peritoneum and lungs are also affected by CRC dissemination [2].

The current management of unresectable metastatic CRC consists of systemic chemotherapy involving various agents, alone or in combination. The choice of therapy is based on several factors, namely the performance status (PS) of patients and the goals of treatment. Also, clinical management of metastatic disease is an example of a multidisciplinary task involving oncologists, surgeons and radiologists. More effective therapeutic regimens together with improved surgical and radiological procedures have nowadays significantly increased stage IV patient's survival rates. Standard treatment of metastatic CRC is based on the use of fluoropyrimidines (fluorouracil or capecitabine), either in monotherapy or combined with oxaliplatin/irinotecan and targeted drugs (bevacizumab, cetuximab, panitumumab, regorafenib and aflibercept). In patients with good PS and without contraindications, a combination therapy is recommended, whereas a monotherapy should be preferred for elderly patients or those with significant comorbidities or in poor clinical condition [3]. Nevertheless, and despite the important improvements in mCRC therapeutic strategies, response rates are still low (30-60\%), meaning that a high number of patients will not benefit from the therapy, but will suffer toxicity-related side effects.

Response to chemotherapy is monitored using imaging, usually by computed tomography (CT) scans, performed regularly and usually every 12 weeks during the course of a given therapy. Disease progression should be confirmed with radiographic testing (eg, CT scan) 
or a biopsy before changing treatment. Morphologic measurement of change in tumor size helps assess therapeutic effectiveness by the use of the Response Evaluation Criteria in Solid Tumors (RECIST) and their modified criteria (version 1.1) during cytotoxic chemotherapy. CT scan data can be quantified and processed to provide accurate and reliable anatomic information about not only tumor shrinkage or growth but also progression of disease by identifying either growth in existing lesions or the development of new lesions. However, there are limitations in the evaluation of tumor response when employing conventional criteria alone, like in detection of peritoneal tumor implants, soft hypodense tissue material associated with chemotherapy, or to adequately correlate with clinical outcomes when assessing treatments that operate via other mechanisms different from tumor size, including molecular targeted treatments and immunotherapies [4]. In addition, imaging methods cannot be repeatedly performed due to risks for patient health derived from radiation overexposure. In a new era of molecular-targeted cancer therapies, the need of more accurate, specific and early response-assessment methods continuously increases. Early predictive markers such as KRAS mutation status have shown to be effective for therapy-based patient stratification, and biomarkers like carcinoembrionic antigen (CEA) are also an indicative tool for treatment effectiveness in mCRC patients, but none of them are conclusive as markers for therapy monitoring.

Metastatic spreading is tightly associated with the propagation of aggressive tumor cells, which successfully escape primary tumors reaching the bloodstream and thus any virtual secondary location [5]. The detection of these circulating tumor cells (CTCs) has demonstrated a clinical prognostic value in patients bearing metastatic tumors, showing reduced survival rates when the number of CTCs met a certain threshold. Using the CellSearch system (Veridex), the unique platform CTC enumeration cleared to date by the American Food and Drug Administration (FDA), it has been reported that the presence of 3 or more CTCs per $7.5 \mathrm{~mL}$ of blood in $\mathrm{mCRC}$ patients predicted poor patient outcome at baseline, e.g., before treatment [6]. Apart from the CellSearch system, other approaches like density gradient centrifugation, filtration or the more recent inertial focusing separation or dean flow fractionation methods, have been proposed as alternatives for CTC isolation from blood [7]. In addition, changes on CTC numbers along treatment have shown a correlation with therapy effectiveness [8]. Thus, CTC analysis represents an attractive candidate for liquid biopsy in cancer, although the immediate future challenge relies on whether treatment decision-making should be based on CTC levels [9]. 


\section{Preliminary clinical studies:}

In a previous work [10], we reported the identification of PrediCTC, a multimarker CTC detection panel that effectively predicted patient outcome before treatment, and provided information about early therapy effectiveness. This multimarker model accurately detected CTC in metastatic CRC ( $\mathrm{mCRC}$ ) patients, based on expression levels of a six-gene panel of tissue specific and Epithelial to Mesenchymal Transition (EMT)-related markers (GAPDH, VIL1, CLU, TIMP1, LOXL3 and ZEB2), and CD45 as an indicator of unspecific cell isolation. This model effectively predicted prognosis, but more importantly it demonstrated an early predictive clinical utility, with an increased sensibility when compared to routine clinical imaging methods. This initial study was conducted in a total of $50 \mathrm{mCRC}$ patients recruited at the University Clinical Hospital of Santiago de Compostela, with the presence of measurable Stage IV CRC, PS equal or less than 2 (based on the Eastern Oncology Cooperative Group (ECOG) scale), and at initiation of a first systemic chemotherapy line, as inclusion criteria. After EpCAM-based CTC immunoisolation and RNA extraction, gene expression levels of CTC-markers, accurately classified patients with high or low levels of CTC, showing a prognostic value. PrediCTC also demonstrated utility to assess therapy response after only one cycle of chemotherapy, with a mean progression-free survival (PFS) of 12.70 months for responders vs. 6.75 months for non-responders $(p=0.004)$, and an overall survival (OS) of 24.26 vs. 13.05 months $(p=0.007)$. These results were further confirmed in an independent prospective multicenter study with $43 \mathrm{mCRC}$ patients recruited in four hospitals from the Galician health system (SERGAS). Briefly, PrediCTC demonstrated an improved sensibility at detection of patient responders after only one cycle of chemotherapy compared to CT-Scan performed at third cycle of therapy (60\% PrediCTC versus $17 \%$ CT scan), in detriment to a reduction in specificity from $98 \%$ by CT scan to $90 \%$ with PrediCTC. This mainly resulted in an improved ability to identify real non-responders (86\% negative predictive value), which is a major clinical necessity for the oncologists in order to decide moving from a non-effective treatment towards a potentially useful therapy as soon as after one cycle of chemotherapy. Overall, and in addition to the accessibility of liquid biopsy and of the clinical information on patient responsiveness, when compared to standard CT scan performed after three cycles of chemotherapy, PrediCTC demonstrated an improved efficiency ( $82 \%$ by PrediCTC versus $77 \%$ by CT-Scan) (Figure $1 ;[11]$ ). 


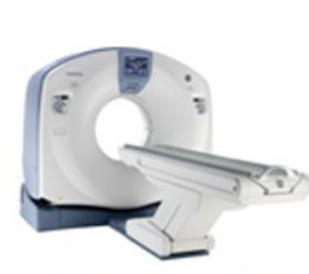

Staging CT

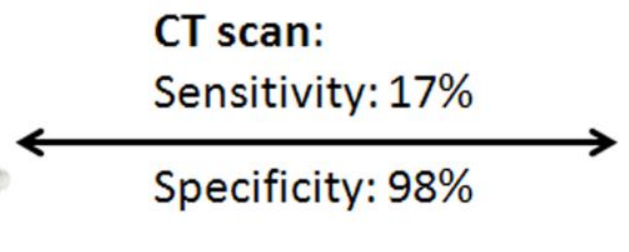

CT scan:
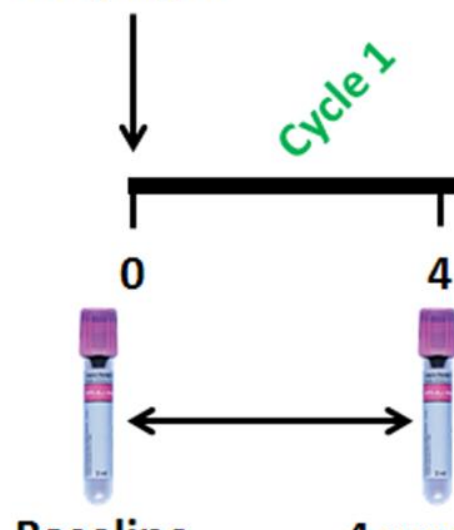

Baseline

PredictC
0
48
8
weeks of treatment

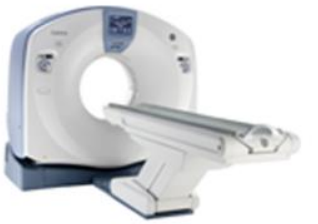

CT scan

12

16

Figure 1: Schema of PrediCTC workflow compared to CT-Scan: while CT-Scan monitors patient response after the third cycle of chemotherapy, PrediCTC evaluates the responsiveness of the therapy after the first cycle of treatment. In addition to the early assessment and the accessibility of the blood sample, PrediCTC results in an improved sensibility compared to CT-Scan, with an affordable reduction in specificity.

\section{Objective of the study:}

These preliminary studies suggested a potential clinical utility of PrediCTC for early therapy response monitoring in advanced CRC patients, with an improved sensibility compared to standard CT scan. We thus aimed to design and develop a technology transfer strategy to define the market opportunity for an eventual implementation of PrediCTC in the clinical practice. This included the definition of the regulatory framework, the analysis of the regulatory roadmap needed for CE mark, a benchmarking study, the design of a product development strategy, a revision of intellectual property (IP), a cost-effectiveness study and an expert panel consultation.

\section{Definition of the regulatory framework for PrediCTC:}

The analysis of the regulatory requirements for this technology determined that PrediCTC is classified as an in vitro diagnostic (IVD) kit as per the In vitro Diagnostic Medical Devices 
Directive 98/79/EC (IVDD), that must follow an autocertificate evaluation for CE mark according to a declaration of conformity. This must be accompanied by the technical file including a complete description of the product, all the documentation related to the quality system, a risk analysis, and the corresponding reports describing the evaluation of performance of the technology, its stability, labelling and instructions for use. In addition, the manufacturer must fulfil the principles of quality guarantee including the organization structure and responsibilities, the adequate processes of fabrication and control of the quality of the production, and the appropriate implementation of the quality system controls. This quality system must be validated by a notified body.

\section{Benchmarking analysis of PrediCTC technology:}

Two main technologies have been identified as similar IVD products approved for commercialisation and potential competitors for PrediCTC: CellSearch Test from Veridex, mentioned above, and AdnaTest from Qiagen. The CellSearch Test (Janssen Diagnostics, LLC; https://www.cellsearchctc.com) is the first and only clinically validated, FDA-cleared test for capturing and enumerating CTCs to help inform clinical decision-making. The evaluation of CTCs by CellSearch allows patient prognosis assessment and is predictive of progression-free survival and overall survival. CellSearch is a semi-automated technology that combines immunoisolation of EpCAM positive epithelial cells from peripheral blood, with the enumeration of circulating tumor cells of epithelial origin (CD45-, EpCAM+, and cytokeratins $8,18+$, and/or 19+).

Although the relative automatization level of sample processing and the accessibility to reproducible interpretation of data permits a reasonable degree of standardization of studies for clinical validation, the strict criteria for CTC definition and the absence of a product actualization strategy since the first studies in advanced breast [12], colorectal (7) and prostate [13] cancer and its commercialisation almost ten years ago, is limiting the use of this technology in the clinical routine.

AdnaTest Colon Cancer (http://www.qiagen.com) is a CE marked product for the detection of CTCs in colorectal cancer patients. Similar to PrediCTC, AdnaTest combines a first step of in vitro CTC enrichment from cancer patients' blood samples using magnetic bead conjugated antibodies optimized for this cancer type, and a second step where the isolated mRNA is transcribed into cDNA that can be amplified in a following multiplex-PCR detection 
step, analysing the tumor associated gene expression of a variety of relevant tumor markers. Compared to CellSearch, the combination of a variety of selection markers for CTC enrichment (tumor-associated antibodies) and a set of molecular tumor markers (EpCAM, CEA and EGFR) improves the sensibility of CTC detection and quantification. Particularly, this allows taking into account CTCs heterogeneity, which prevents for false negative and positive results due to either marker expression in normal blood cells or gene expression changes during chemotherapy. Also similar to PrediCTC, Adnatest includes a first kit for the isolation of CTCs and the subsequent extraction of mRNA from human whole blood, and second RT-PCR kit for detection of colon cancer-associated gene expression in enriched tumor cells. To date, limited studies with clinical evidences have demonstrated the utility of AdnaTest technology at the clinical setting [14].

\section{Product development strategy for PrediCTC:}

The development plan for PrediCTC has been defined following the international standards for quality management systems for medical devices (ISO 13485) and for performance evaluation of in vitro diagnostic medical devices (ISO 13612). First, the design of the PrediCTC diagnostic IVD kit, including the physical prototype and the clinical protocol for the processing and management of the samples, was preferably defined as a "minimumm viable kit (MVK)" containing all those components considered as "know how"; the remaining elements provided out of the kit, to facilitate the manufacture, reduce costs and diminish stability risks.

Second, the PrediCTC diagnostic IVD kit must be validated through a scientific validity and a performance evaluation (Figure 2). The scientific validity containing all the information on the validity of CTC evaluation for the follow-up of treatment of patients with metastatic colorectal cancer and on the limitations of PrediCTC, includes information from equivalent IVD products as CellSearch and ADNAtest, from the literature and opinion documents, and from clinical data obtained with PrediCTC. The performance evaluation corresponding to a new test must follow an analytical and a clinical evaluation. The analytical evaluation demonstrates the efficacy of the technology and includes data on sensibility and specificity, compared to the CT scan as standard reference. The clinical evaluation aims to evaluate the efficacy of PrediCTC in clinical practice conditions. This includes the target population, the characterisation and processing of the samples, the characterisation of the analyte and its detection, the reactives and the equipment. For this, a new multicentric, prospective, 
observational and longitudinal study in a 150-300 patient population performed in 3-6 months should provide conclusive data on overall percent assessment. Finally, the estimated timing and costs for the development plan are one year and approximately $300 \mathrm{k}$ euros, including registry of CE mark and licence.

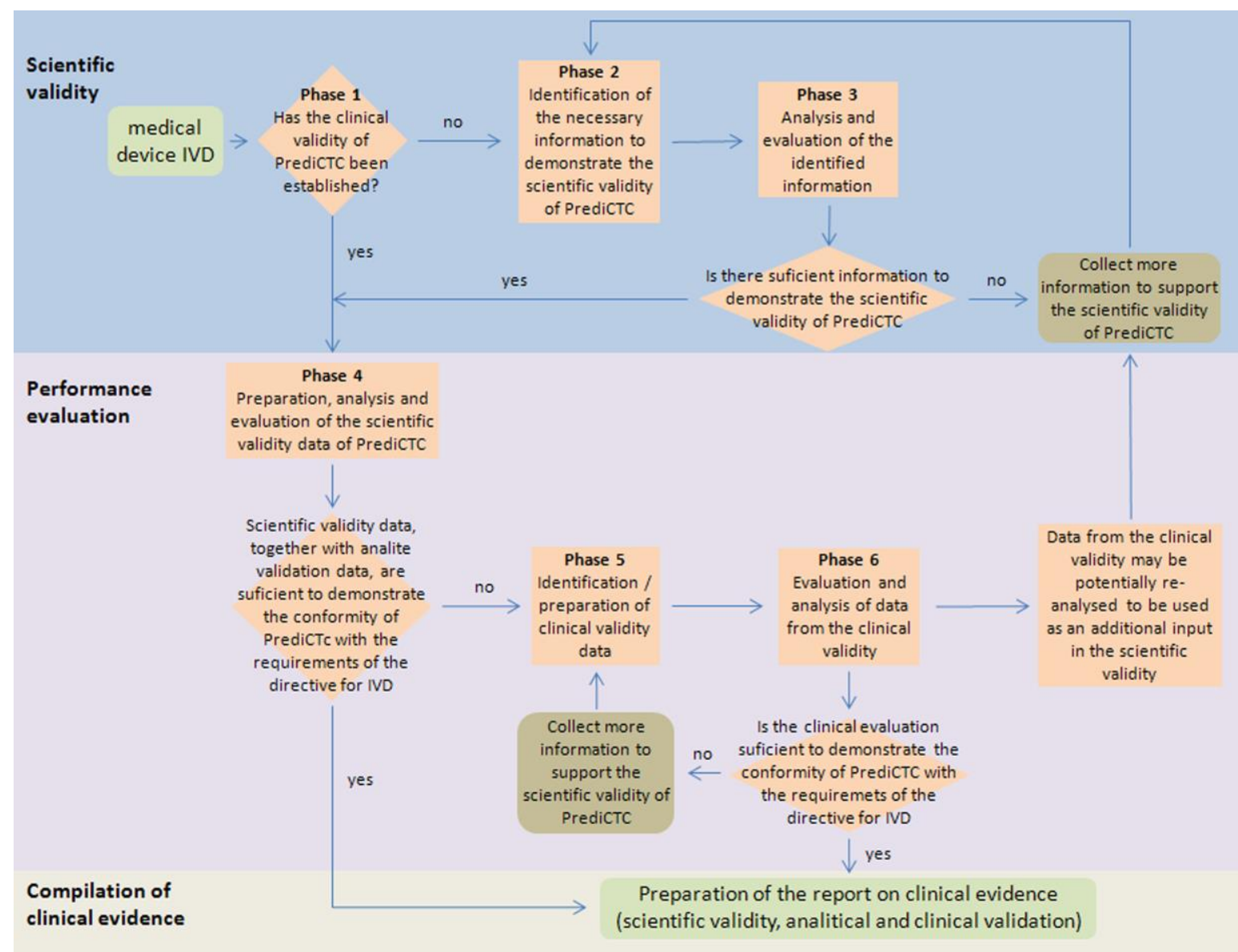

Figure 2: Performance evaluation of PrediCTC technology.

\section{Intellectual Property analysis:}

PCT/EP2015/056649 application provides a panel of biomarkers with predictive value for therapy effectiveness in patients suffering from metastatic colorectal cancer. In particular, this patent application provides a method for determining progression free survival and overall survival in response to therapy in $\mathrm{mCRC}$ patients by assessing the expression level of at least one gene selected from the group consisting of LOXL3, ZEB2, GAPDH, VIL1, TIMP1, CLU and TLN1 in the circulating tumor cells of a blood sample taken from the patient (see CLAIMS in Annex A). 
In the international phase of the PCT, the Written Opinion raised a number of objections, in particular a unity of invention objection as well as a lack of inventive step objection to all those claims linked by the presence of LOXL3, with the exception of those claims referring to the specific combination of the following seven genes: GAPDH, VIL1, TIMP1, CLU, TLN1, LOXL3 and ZEB2. Therefore, a priori, the protection conferred by PCT/EP2015/056649 is limited to the combination of GAPDH, VIL1, TIMP1, CLU, TLN1, LOXL3 and ZEB2. Such protection is certainly limited.

In addition, PCT/EP2015/056649 is silent about the possibility of using kits or devices for implementing the method disclosed therein. Lastly, in case these limitations can be overcome, the commercialization opportunity of a PrediCTC kit or device must be determined by an evaluation of any possible intellectual property hurdles to said commercialization through a freedom to operate analysis.

\section{Cost-effectiveness evaluation:}

This section on economic evaluation summarizes the estimated cost-effectiveness of PrediCTC, as a multimarker panel from circulating tumor cells developed to assess the chemotherapy response, versus CT scan, in the follow up of first-line treatment response in patients with un-resectable $\mathrm{mCRC}$, described in [15].

Based on Barbazan et al., 2014 and ESMO (European Society for Medical Oncology) guidelines, a Markov model has been developed with transition probabilities being established by free progression survival and overall survival results from the main clinical trials for the different treatments received by $\mathrm{mCRC}$ patients, together with utilities and adverse events rates [15]. As well, direct and indirect resources use and unitary cost data were obtained from published studies, drugs technical files, and regional health systems' prices.

Base case analysis showed savings in all costs, and probabilistic sensitivity analysis results confirmed the data from the base case scenario: PrediCTC is more efficient than CT Scan with a probability of $91.20 \%$ to be cost-effective at a $30,000 € / Q A L Y$ threshold. CT Scan option achieves a higher utility because the model is prepared to assign lower utilities to patients as they progress through chemotherapy lines. Finally, from the Spanish societal perspective PrediCTC results a cost-effectiveness option as it allows identifying patients that 
are not getting benefits from therapy in $\mathrm{MCRC}$ and therefore to avoid side effects and costs in those who are non-responders.

\section{Expert panel consultation:}

We finally explored the viability of PrediCTC in the clinical practice by exposing PrediCTC technology and its preliminary clinical data to a panel of expert Spanish oncologists. Three main concerns were identified by the experts for the implementation of PrediCTC in the clinical routine at the medical oncology departments in hospitals of the Spanish healthcare system: first, the likelihood that PrediCTC end up by substituting CT scan in the follow-up of $\mathrm{mCRC}$ patients, at least for the first line of chemotherapy, is not realistic as the images of the tumor lesions are necessary for the evaluation of an eventual surgical treatment. This would imply a coexistence of PrediCTC and the CT scan, with complementary information, as a more reasonable although less cost-effective scenario for its implementation in the oncology practice. The validation of PrediCTC panel in more advanced CRC therapy lines could open a more favorable scenario where CT scan could be effectively replaced by liquid biopsy, with quality-of-life as main outcome.

Second, the competitive advantage of PrediCTC faced to the two technologies already in the market, CellSearch and AdnaTest, should be based not only in the real-time quantification of the CTCs in MCRC patients allowing the assessment of therapy response, but on additional information providing, for example, clues for the selection of the alternative and more efficient therapeutic regimen. In addition, the irruption of ctDNA-related technologies, with an apparent more evident applicability in clinical routine, raises a new scenario in which the competitiveness of CTC could be compromised; in this sense, and although the utility of the CTC analysis remains clear and probably the technologies based on ctDNA and CTC ends up being complementary in the clinical setting, so far there are uncertainties [16].

Third, the implementation of PrediCTC in the clinical routine, although favored by the minimally invasive collection of a peripheral blood sample, is limited by their complex and time-consuming processing, with approximately eight hours of a specialized technician for the immunoisolation of CTCs, RNA extraction, pre-amplification and RT-q-PCR for PrediCTC panel and interpretation and report of data. In addition, the number of mCRC 
patients per hospital in Spain does not represent an effective volume for a technology like PrediCTC.

\section{Conclusions:}

PrediCTC represents an attractive example of precision medicine, demonstrating the potential of liquid biopsy in the management of patients in oncology. The competitive advantage of PrediCTC versus the CT scan as the standard technique in monitoring of patients has been demonstrated by the preliminary clinical data in a global cohort of 93 mCRC patients, effectively classifying them in responders and non-responders after only one cycle of chemotherapy, with a minimally invasive blood sample, and with an improved sensibility. The reduction in toxic secondary effects and costs of inefficacious cycles of chemotherapy represent the principal benefits of PrediCTC. The regulatory roadmap is clear and reasonable in terms of costs and time to market. Nevertheless, the limitations in the IP protection and in the implementation in the clinical practice represent two major concerns to effectively translate PrediCTC into the market. A different commercialization strategy, based on know-how and a centralized laboratory, and more advanced third/forth lines of chemotherapy as a different target population where PrediCTC could demonstrate an additional benefit in terms of quality of life, are potential alternatives for the efficient transfer of PrediCTC into the market. 


\section{Compliance with Ethical Standards:}

The authors declare no conflict of interest. Code of Institutional Ethics Committee: 2014/464; informed consent were signed by all patients. 


\section{References:}

[1] R. L. Siegel, K. D. Miller, and A. Jemal, "Cancer statistics, 2016" CA. Cancer J. Clin., vol. 66, no. 1, pp. 7-30, Jan. 2016.

[2] D. Sargent, A. Sobrero, A. Grothey, M. J. O'Connell, M. Buyse, T. Andre, et al., "Evidence for cure by adjuvant therapy in colon cancer: observations based on individual patient data from 20,898 patients on 18 randomized trials." J. Clin. Oncol., vol. 27, no. 6, pp. 872-7, Feb. 2009.

[3] G. Brandi, S. De Lorenzo, M. Nannini, S. Curti, M. Ottone, F. G. Dall'Olio, et al., "Adjuvant chemotherapy for resected colorectal cancer metastases: Literature review and meta-analysis." World J. Gastroenterol., vol. 22, no. 2, pp. 519-33, Jan. 2016.

[4] E. Van Cutsem, H. M. Verheul, P. Flamen, P. Rougier, R. Beets-Tan, R. GlynneJones, et al., "Imaging in Colorectal Cancer: Progress and Challenges for the Clinicians." Cancers (Basel)., vol. 8, no. 9, p. 81, Aug. 2016.

[5] D. Hanahan and R. A. Weinberg, "Hallmarks of cancer: the next generation." Cell, vol. 144, no. 5, pp. 646-74, Mar. 2011.

[6] S. J. Cohen, C. J. Punt, N. Iannotti, B. H. Saidman, K. D. Sabbath, G. Y. Gabrail, et al., "Prognostic significance of circulating tumor cells in patients with metastatic colorectal cancer." Ann. Oncol., vol. 20, no. 7, pp. 1223-1229, Jul. 2009.

[7] B. P. Negin and S. J. Cohen, "Circulating tumor cells in colorectal cancer: past, present, and future challenges." Curr. Treat. Options Oncol., vol. 11, no. 1-2, pp. 113, Jun. 2010.

[8] S. J. Cohen, C. J. Punt, N. Iannotti, B. H. Saidman, K. D. Sabbath, G. Y. Gabrail, et al., "Relationship of Circulating Tumor Cells to Tumor Response, Progression-Free Survival, and Overall Survival in Patients With Metastatic Colorectal Cancer." J. Clin. Oncol., vol. 26, no. 19, pp. 3213-3221, Jul. 2008.

[9] C. Alix-Panabières and K. Pantel, "Circulating tumor cells: liquid biopsy of cancer." Clin. Chem., vol. 59, no. 1, pp. 110-8, Jan. 2013.

[10] J. Barbazan, L Muinelo-Romay, M. Vieito, S. Candamio, A. Diaz-Lopez, A. Cano, et al., "A multimarker panel for circulating tumor cells detection predicts patient outcome and therapy response in metastatic colorectal cancer." Int. J. Cancer, vol. 135, no. 11, pp. 2633-2643, Dec. 2014.

[11] Y. Vidal Insua, J. Camara, E. B. Vazquez, A. Fernandez, F. Rivera, M. J. V. V. Silva, et al., "Predicting outcome and therapy response in $\mathrm{mCRC}$ patients using an indirect method for CTCs detection by a multigene expression panel: a multicentric prospective validation study." Int. J. Mol. Sci., vol. 18, no. 6, Jun 2017.

[12] D. F. Hayes, M. Cristofanilli, G. T. Budd, M. J. Ellis, A. Stopeck, M. C. Miller, et al., 
"Circulating Tumor Cells at Each Follow-up Time Point during Therapy of Metastatic Breast Cancer Patients Predict Progression-Free and Overall Survival." Clin. Cancer Res., vol. 12, no. 14, pp. 4218-4224, Jul. 2006.

[13] J. S. de Bono, H. I. Scher, R. B. Montgomery, C. Parker, M. C. Miller, H. Tissing, et al., "Circulating tumor cells predict survival benefit from treatment in metastatic castration-resistant prostate cancer." Clin. Cancer Res., vol. 14, no. 19, pp. 6302-9, Oct. 2008.

[14] S. Lankiewicz, S. Zimmermann, C. Hollmann, T. Hillemann, and T. F. Greten, "Circulating tumour cells as a predictive factor for response to systemic chemotherapy in patients with advanced colorectal cancer." Mol. Oncol., vol. 2, no. 4, pp. 349-55, Dec. 2008.

[15] C. Anton, M. Abal, L. Alonso-Alconada, S. Candamio, R. Lopez-Lopez, C. Martin, "Monitoring Treatment Response in Metastasic Colorectal Cancer: Economic Evaluation of PrediCTC® versus CT Scan." Submitted.

[16] C. R. C. Tan, L. Zhou, and W. S. El-Deiry, "Circulating Tumor Cells Versus Circulating Tumor DNA in Colorectal Cancer: Pros and Cons." Curr. Colorectal Cancer Rep., vol. 12, no. 3, pp. 151-161, Jun. 2016. 


\section{Annex A.}

CLAIMS (PCT/EP2015/056649)

1. A method for determining the outcome of a subject suffering from metastatic colorectal cancer (mCRC) and/or the effectiveness of a therapy administered to said subject that comprises the steps of:

a) taking a first follow-up blood sample from the subject after having been administered at least one therapy cycle;

b) assessing the expression level of at least one gene selected from the set of genes of the group of LOXL3, ZEB2, GAPDH, VIL1, TIMP1, CLU and TLN1 in the CTCs of the blood sample;

c) classifying the expression level of the selected gene, or of each one of the set of genes selected for being analysed, in the CTCs of the blood sample as "high" when it is higher than a reference cutoff value of the expression level of said gene and "low" when it is equal or lower than said reference cutoff value;

d) classifying the blood sample as

i. "low-CTC", if the expression level of the selected gene in CTCs is low or, when more than one gene of the above mentioned set of genes is being analysed, if the expression level in CTCs of more than a half of said analysed genes is low;

ii. "high-CTC", if the expression level of the selected gene in CTCs is high or, when more than one gene of the above mentioned set of genes is being analysed, if the expression level in CTCs of at least one half of said analysed genes is high;

e) assessing the effectiveness of the administered therapy by classifying the subject as:

i. "non responder" to the therapy, if the subject is "high-CTC" for the analysed follow-up blood sample, and

ii. "responder" to the therapy, if the subject is "low-CTC" for the analysed follow-up blood sample;

f) optionally, taking a second or additional follow-up blood samples from the subject after having been administered a therapy cycle subsequent to the therapy cycle already administered when the first follow-up sample was taken and repeating steps b) to e) with said second or subsequent follow-up sample to confirm the previous assessment of therapy effectiveness;

g) additionally, or alternatively to steps e) and f), predicting the outcome of the subject at a time point of therapy course by classifying the subject as a "high risk" subject when 
the blood sample taken at that time point is "high-CTC", and as a "low risk" subject when the blood sample taken at that time point is "low-CTC".

2. The method according to claim 1, wherein the expression level of at least two, three, four, five, six or all the seven genes of the group of LOXL3, ZEB2, GAPDH, VIL1, TIMP1, CLU and TLN1 is determined and classified in steps a) and b) and used for classifying the blood sample and the patients in step c) and d) and e) and, when carried out, in step e) and/or f). 3. The method according to claim 1 or 2, wherein LOXL3 and/or ZEB2 is selected for determining and classifying its expression level in steps a) and b) and used for classifying the blood sample and the patients in step c) and d) and, optionally, in step e) and, when carried out, in step e) and/or f).

4. The method according to claim 2 and 3, wherein the expression level of at least the two genes LOXL3 and VIL1 is determined and classified in steps a) and b) and used for classifying the blood sample and the patients in step c) and d) and, when carried out, in step e) and/or f).

5. The method according to claim 4, wherein the expression level of at least the three genes LOXL3, VIL1 and CLU is determined and classified in steps a) and b) and used for classifying the blood sample and the patients in step c) and d) and, when carried out, in step e) and/or f).

6. The method according to claim 5, wherein the expression level of at least the four genes LOXL3, VIL1, CLU and GAPDH is determined and classified in steps a) and b) and used for classifying the blood sample and the patients in step c) and d) and, when carried out, in step e) and/or f).

7. The method according to any one of claims 1 to 3 , wherein six genes of the group of GAPDH, VIL1, TIMP1, CLU, TLN1, LOXL3 and ZEB2 are selected for carrying out the steps of the method and the six genes are: GAPDH, VIL1, TIMP1, CLU, LOXL3 and ZEB2, or GAPDH, VIL1, CLU, TLN1, LOXL3 and ZEB2, or, GAPDH, VIL1, TIMP1, TLN1, LOXL3 and ZEB2, and wherein the blood sample to be classified in step c) is classified as "low-CTC" when the expression level of at least four genes of the set of analysed genes is low, and "high-CTC" when the expression level of three or more genes of the set of analysed genes is high.

8. The method according to any one of claims 1 to 3 , wherein the seven genes of the group of GAPDH, VIL1, TIMP1, CLU, TLN1, LOXL3 and ZEB2 are selected for carrying out the steps of the method, and wherein the blood sample to be classified in step c) is classified as "low-CTC" when the expression level of at least four genes of the set of analysed genes is 
low, and "high-CTC" when the expression level of three or more genes of the set of analysed genes is high.

9. The method according to any one of claims 1 to 8 , wherein a previous blood sample is taken from the subject before having administered the therapy cycle that has been administered when the first follow-up sample is taken, and steps b) to e) and, optionally, f), and, optionally or alternatively to steps e) and f), step g), are also performed in said previous blood sample.

10. The method according to claim 9 , wherein step $f$ ) of confirmation is carried out when the subject is "low-CTC" for the follow-up blood sample considered in step d) and "high-CTC" for the immediately previous sample.

11. The method according to claim 10, wherein the follow-up sample considered in step d) is the first follow-up blood sample and the immediately previous sample is the baseline sample taken before the start of the therapy.

12. The method according to any one of claims 9 to 11 , wherein step g) is carried out for predicting the outcome of the patient before therapy, on a blood sample which is the baseline sample taken before the start of the therapy.

13. The method according to any one of claims 1 to 11 , wherein the administered therapy to be assessed is chemotherapy

14. The method according to claim 13, wherein the chemotherapy to be assessed comprised the administration of at least one fluoropyrimidine (fluorouracil or capecitabine) alone or in combination with oxaliplatin or irinotecan and/or with anti-EGFR or anti-VEGF antibodies.

15. The method according to claim 13 or 14, wherein the first follow-up blood sample is taken before therapy cycle 2 or 4 weeks after the start of therapy.

16. The method according to claim 14 or 15 , wherein step f) is carried out and a second follow-up blood sample is taken before therapy cycle 3 or 16 weeks after the start of therapy. 17. The method according to any one of the preceding claims, wherein the expression level of each analysed gene is normalized with regard to the expression level of a reference gene. 18. The method according to claim 17, wherein the reference gene is CD45.

19. The method according to any one of the preceding claims, wherein the blood samples are enriched in CTCs by using a immunoaffinity technique based on anti-EpCAM antibodies. 20. The method according to any one of the preceding claims, wherein the expression level of each gene in each sample is assessed by quantifying the level of its corresponding mRNA in said sample. 
21. The method according to any one of the preceding claims, wherein each reference cutoff value that is used in step $c$ ) has been determined in a statistical study with $\mathrm{mCRC}$ patients as the $75 \%$ percentile value of the expression level of the corresponding gene at the time point of the therapy course wherein the blood sample to be classified has been taken.

22. The method according to any one of the preceding claims, wherein the assessment is complemented with the results of an imaging technique such a CT colonography and/or with a monitoring technique based on the determination of a biomarker in serum such as CA-125 or CEA. 